\title{
Crustáceos decápodos associados a esponjas no litoral do Estado do Ceará, Brasil
}

\author{
Luis E. A. Bezerra ${ }^{1}$ \& Petrônio A. Coelho ${ }^{2}$ \\ ${ }^{1}$ Autor correspondente. Departamento de Biologia, Centro de Ciências, Universidade Federal do Ceará. Avenida Mister Hull, \\ Campus do Pici, 60455-760 Fortaleza, Ceará, Brasil. E-mail: luiseab@gmail.com \\ 2 Departamento de Oceanografia, Universidade Federal de Pernambuco. Avenida da Arquitetura, Cidade Universitária, \\ 50670-901 Recife, Pernambuco, Brasil. Bolsista de produtividade do CNPq. E-mail: petronio.coelho@bol.com.br
}

\begin{abstract}
Sponge-dwelling crustaceans at Ceará state litoral, Brazil. The aim of this article was to report on the sponge-dwelling crustaceans collected through scuba diving at "Parque Estadual Marinho Pedra da Risca do Meio" Fortaleza, Ceará, Brazil. Nine species of sponges were collected and fourteen crustaceans were identified. The Alpheidae family was the most abundant with six species, followed by the families Mithracidae and Porcellanidae with two species and Palaemonidae, Pilumnidae, Pseudorhombilidae and Xanthidae with one species. The species Nanoplax xanthiformis (A. Milne Edwards, 1880), Synalpheus hemphilli Coutière, 1909, S. minus (Say, 1818), S. sanctithomae Coutière, 1909 and Petrolisthes rosariensis Werding, 1978 have their distribution expanded in the brazilian coast. KEY WORDS. Commensalism; Decapoda; first record.
\end{abstract}

RESUMO. Neste trabalho é apresentada a fauna de crustáceos decápodos associados a esponjas coletadas ao largo da cidade de Fortaleza, Ceará, Brasil. As esponjas foram obtidas através de mergulho autônomo, realizado no Parque Estadual Marinho "Pedra da Risca do Meio". No laboratório, os poríferos foram identificados e os crustáceos retirados e etiquetados de acordo com a esponja em que foram encontrados. Nove espécies de esponjas foram coletadas e 14 espécies de crustáceos identificadas. Os Crustáceos da família Alpheidae foram os mais representativos, com seis espécies, seguidos pelos decápodos das famílias Mithracidae e Porcellanidae, com duas espécies e Palaemonidae, Pilumnidae, Pseudorhombilidae e Xanthidae, com uma espécie. A partir do presente registro, as espécies Nanoplax xanthiformis (A. Milne Edwards, 1880), Synalpheus hemphilli Coutière, 1909, S. minus (Say, 1818), S. sanctithomae Coutière, 1909 e Petrolisthes rosariensis Werding, 1978 têm sua distribuição aumentada no litoral brasileiro.

PALAVRAS-CHAVE. Comensalismo; Decapoda; primeiros registros.

Várias espécies de crustáceos decápodos têm sido reportadas vivendo em associação com outros organismos marinhos, principalmente corais e esponjas (BRUCE 1976). Algumas vivem exclusivamente como comensais, dentre as quais pode-se citar os camarões da família Alpheidae, onde mais de 100 espécies de Synalpheus Bate, 1888 foram descritas vivendo exclusivamente associadas a invertebrados sésseis, particularmente esponjas e crinóides (Ríos \& Dufry 1999)

Com relação às esponjas, uma fauna variada pode ser encontrada em seus canais interiores e em sua superfície. Nesta, os crustáceos decápodos são, há muito tempo, bem conhecidos como ocorrendo neste ambiente, tendo BaLSS (1956) feito um levantamento da literatura sobre este tema até aquela época. Nos anos seguintes foram publicados numerosos trabalhos fazendo referência a este fato, porém não foram encontradas citações para o Estado do Ceará. Assim, o presente trabalho reporta a fauna de crustáceos decápodos encontrada associada a esponjas, coletadas em uma área de conservação ambiental na costa cearense.

\section{MATERIAL E MÉTODOS}

As esponjas foram coletadas por mergulho autônomo ("scuba diving") no Parque Estadual Marinho "Pedra da Risca do Meio", ao largo de Fortaleza, Ceará, nos dias 14 e 15 de julho de 2004, entre 20 e $22 \mathrm{~m}$ de profundidade (Fig. 1). Os espécimes foram dissecados para extração dos organismos comensais, sendo os crustáceos decápodos selecionados para o presente estudo. As esponjas foram identificadas por E. Hajdu, do Museu Nacional da Universidade Federal do Rio de Janeiro. Os crustáceos foram identificados pelos presentes autores e estão depositados na Coleção Carcinológica do Departamento de Oceanografia da Universidade Federal de Pernambuco e na Coleção Carcinológica do Instituto de Ciências do Mar (LABOMAR) da Universidade Federal do Ceará. O Parque Estadual Marinho da Pedra da Risca do Meio foi criado através da Lei Estadual No 12.717 de 05 de Setembro de 1997. É a única Unidade de Conservação Marinha do Estado do Ceará, com 


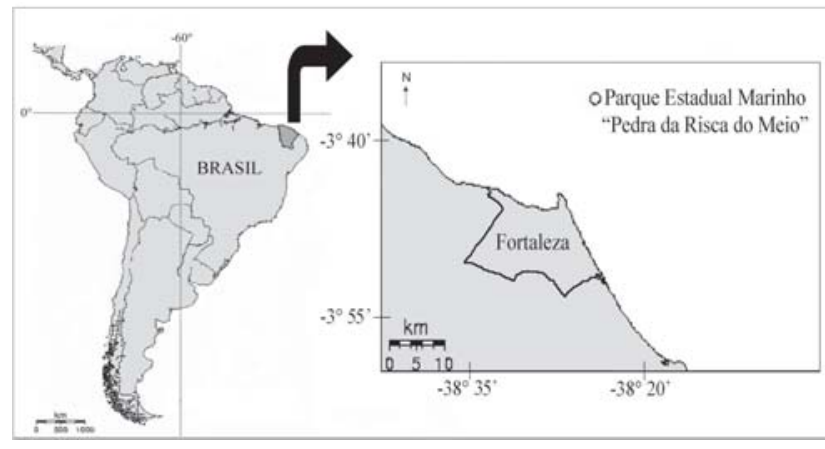

Figura 1. Localização da área de coleta, ao largo da cidade de Fortaleza, Ceará.

uma área de $33,20 \mathrm{~km}^{2}$, distante 10 milhas náuticas (aproximadamente $18,5 \mathrm{~km}$ ) do Porto do Mucuripe, em Fortaleza, na direção $60^{\circ} \mathrm{NE}$ (sessenta graus nordeste). O Parque Marinho está delimitado pelas seguintes coordenadas geográficas: A: $3^{\circ} 33^{\prime} 48^{\prime \prime}$ S e $38^{\circ} 26^{\prime} 00^{\prime \prime} \mathrm{W}$; B: $3^{\circ} 36^{\prime} 00^{\prime \prime} \mathrm{S}$ e $38^{\circ} 26^{\prime} 00^{\prime \prime} \mathrm{W}$; C: $3^{\circ} 36^{\prime} 00^{\prime \prime}$ S e $38^{\circ} 21^{\prime} 00^{\prime \prime} \mathrm{W}$; D: $3^{\circ} 33^{\prime} 48^{\prime \prime} \mathrm{S}$ e $38^{\circ} 21^{\prime} 00^{\prime \prime} \mathrm{W}$.

\section{RESULTADOS E DISCUSSÃO}

Todas os poríferos coletados apresentavam crustáceos em seus canais interiores. Foram encontradas 14 espécies de decápodos associados a esponjas, pertencentes a sete famílias. Além dos crustáceos, também foram encontrados poliquetas e equinodermas. A lista dos crustáceos coletados e os hospedeiros em que se encontravam está apresentada na tabela I. A classificação taxonômica adotada segue a proposta por MARTIN \& DAVIS (2001).

Balss (1956) menciona que naquela época se conheciam várias espécies de decápodos comensais de esponjas pertencentes às famílias Alpheidae, Palaemonidae (da subfamília Pontoniinae), Stenopodidae, Axiidae, Porcellanidae e Pilumnidae. Estes organismos tinham sido encontrados em todos os oceanos.

No Nordeste do Brasil, Rocha et al. (2000) mencionaram alguns organismos vivendo em esponjas tubulares na plataforma continental do Rio Grande do Norte e da Paraíba, incluindo os crustáceos decápodos das famílias Alpheidae, Mithracidae, Porcellanidae, Pilumnidae e Stenopodidae. Na presente pesquisa, foram encontrados representantes das famílias Alpheidae, Mithracidae, Palaemonidae, Pilumnidae, Porcellanidae, Pseudorhombilidae e Xanthidae.

Os Alpheidae estiveram bem representados nas esponjas estudadas. O gênero Synalpheus possui várias espécies habitantes de esponjas, assim, CHRISTOFFersen (1979) indica como sendo encontradas em esponjas as seguintes espécies que ocorrem no Brasil: S. brevicarpus, S. brooksi Coutière, 1909, S. fritzmuelleri Coutière, 1909, S. hemphilli, S. longicarpus (Herrick, 1891), S. minus, S. santithomae e $S$. townsendi. Algumas espécies de Synalpheus estão registradas para mais de uma espécie de esponja. A identificação das espécies tem se mostrado difícil e, segundo Dufry (1996), existem espécies crípticas que são específicas quanto ao hospedeiro e que podem ser distinguidas entre si por caracteres morfológicos, inclusive a coloração do animal quando vivo e pelo genótipo. Seria necessário, portanto, reexaminar os casos em que uma espécie de Synalpheus foi assinalada em mais de uma espécie de esponja. Em muitos casos, no entanto, não é possível encontrar informações minuciosas sobre o habitat das amostras.

Não é conhecida, na fauna brasileira, a ocorrência de Mithracidae comensal de esponjas, embora seja quase universal a presença de esponjas vivendo sobre a carapaça destes crustáceos, sendo utilizadas como forma de disfarce. Observar que Mithraculus forceps, foi encontrada associada a três espécies de esponjas distintas. Este Mithracidae, no entanto, é uma das poucas espécies que não se disfarçam com esponjas, o que torna totalmente inesperado o presente achado.

O camarão Palaemonidae Periclimenaeus bermudensis é considerado comensal por Ramos-Porto \& Coelho (1998) e Holthuis (1951) assinala sua ocorrência no interior de esponjas. Também P. perlatus (Boone, 1930) vive em pares dentro de cavidades de esponjas (Ramos-Porto \& Coelho 1998), e P. pearsei (Schmitt, 1932) é encontrado na esponja Spongia officinalis (Linnaeus, 1759) (СHACE 1972); outras espécies, igualmente comensais, vivem associadas a outros hospedeiros, ou, até mesmo, a vários hospedeiros.

Os caranguejos da família Pilumnidae, particularmente aqueles do gênero Pilumnus Leach, 1815, são encontrados numa variedade de tipos de fundo, inclusive sobre esponjas, não parecendo especialmente adaptados ao modo de vida comensal (Melo 1996).

Os Porcellanidae do gênero Petrolisthes Stimpson, 1858 são encontrados em cavidades associadas ao ambiente rochoso; $P$. rosariensis, no entanto, é conhecida de arrecifes de coral e de algas calcárias (Veloso \& Melo 1993, Veloso 1998) e sua presença nas esponjas não foi registrada anteriormente, embora, certamente, se deva à existência de depressões e orifícios onde poderia se ocultar.

O Pseudorhombilidae Nanoplax xanthiformis não parece adaptado à vida de comensalismo, sendo sua presença provavelmente ocasional.

O Xanthidae Xanthodius denticulatus, embora conhecido de uma variedade de tipos de fundo, não tem sido encontrado como comensal de esponjas.

Este trabalho estende a distribuição geográfica das espécies Nanoplax xanthiformis, Petrolisthes rosariensis, Synalpheus hemphilli, S. minus e $S$. sanctithomae no litoral brasileiro. $N$. xanthiformis tinha distribuição conhecida, até o momento, para os Estados do Amapá (Barreto et al. 1993), Pará (Barreto et al. 1993) e Rio de Janeiro (Rathbun 1898). P. rosariensis ocorria da Paraíba até a Bahia (Veloso \& Melo 1993, Coelho-Santos \& CoeLHo 1994). Quanto aos alfeideos, só havia um registro prévio de S. hemphili na costa brasileira, no Estado da Bahia (CHRISTOFFerSeN 1979). Synalpheus minus ocorria nos penedos de São Pedro e São 
Tabela I. Espécies de crustáceos comensais com as respectivas esponjas em que foram encontradas, coletadas no Parque Estadual Marinho "Pedra da Risca do Meio", Fortaleza, Ceará.

\begin{tabular}{|c|c|c|}
\hline Família & Espécie & Esponja \\
\hline Palaemonidae & Periclimenaeus bermudensis (Armstrong, 1940) & Aplysina fistularis (Pallas, 1766) \\
\hline \multirow[t]{6}{*}{ Alpheidae } & Synalpheus brevicarpus (Herrick, 1891)* & Ircinia felix (Duchassaing \& Michelotti, 1864) \\
\hline & Synalpheus filidigitus Armstrong, 1949 & Aiolochroia crassa (Hyatt, 1875) \\
\hline & Synalpheus hemphilli Coutière, 1909 & Callyspongia vaginalis (Lamarck, 1818) \\
\hline & Synalpheus minus (Say, 1818) & Callyspongia vaginalis (Lamarck, 1818) \\
\hline & Synalpheus sanctithomae Coutière, 1909 & $\begin{array}{l}\text { Ircinia strobilina (Lamarck, 1816) e Agelas clathrodes } \\
\text { (Schmidt, 1870) }\end{array}$ \\
\hline & Synalpheus townsendi Coutière, 1909 & Monanchora arbuscula (Duchassaing \& Michelotti, 1864) \\
\hline \multirow[t]{2}{*}{ Porcellanidae } & Petrolisthes galathinus (Bosc, 1808/02) & $\begin{array}{l}\text { Agelas díspar (Duchassaing \& Michelotti, 1864), } \\
\text { Dragmacidon reticulatus (Ridley \& Dendy, 1886) e } \\
\text { Callyspongia vaginalis (Lamarck, 1818) }\end{array}$ \\
\hline & Petrolisthes rosariensis Werding, 1978 & Callyspongia vaginalis (Lamarck, 1818) \\
\hline \multirow[t]{2}{*}{ Mithracidae } & Leptopisa setirostris (Stimpson, 1871) & Monanchora arbuscula (Duchassaing \& Michelotti, 1864) \\
\hline & Mithraculus forceps (A. Milne Edwards, 1875) & $\begin{array}{l}\text { Dragmacidon reticulatus (Ridley \& Dendy, 1886), } \\
\text { Callyspongia vaginalis (Lamarck, 1818) e Ircinia felix } \\
\text { (Duchassaing \& Michelotti, 1864) }\end{array}$ \\
\hline Pilumnidae & Pilumnus quoyi Milne Edwards, 1834 & Ircinia felix (Duchassaing \& Michelotti, 1864) \\
\hline Pseudorhombilidae & Nanoplax xanthiformis (A. Milne Edwards, 1880) & Agelas clathrodes (Schmidt, 1870) \\
\hline Xanthidae & Xanthodius denticulatus (White, 1847) & Dragmacidon reticulatus (Ridley \& Dendy, 1886) \\
\hline
\end{tabular}

* Parasitado por Bopyridae.

Paulo, Fernando de Noronha e nos Estados de Pernambuco, Alagoas e Bahia (Bate 1888, Rathbun 1900, Coelho et al. 2002), enquanto $S$. sanctithomae era conhecido para o Atol das Rocas e nos Estados de Pernambuco e Bahia (Christoffersen 1979). Entretanto, P.A. Coelho-Filho, em sua tese de doutorado, defendida em 2002, reportou a ocorrência dessa espécie para o Ceará.

\section{AGRADECIMENTOS}

A Tito M.C. Lotufo (Departamento de Engenharia de Pesca, UFC) e a Paula C. Jimenez por tornarem possível a coleta das esponjas; Eduardo Hajdu (Museu Nacional, UFRJ), pela identificação das esponjas; Wilson Franklin Júnior (Labomar, UFC), pela ajuda na coleta dos crustáceos e Alexandre O. de Almeida (UESC), pelas sugestões e revisão do manuscrito.

\section{REFERÊNCIAS BIBLIOGRÁFICAS}

BaLss, H. 1956. Decapoda als Comensalen, p. 1405-1417. In: H.G. BRonNs (Ed.). Klassen und Ordnungen des Tierreichs. Fünfter Bamd, I. Abteilung, 7. Buch, 11. Lieferung. Leipzig, Akademische Verlagsgesellschaft Geest \& Portig.

Barreto, A.V.; P.A. Coelho \& M. Ramos-Porto. 1993. Distribuição dos Brachyura (Crustacea, Decapoda) coletados na plataforma continental do Norte e Nordeste do Brasil. Revista Brasileira de Zoologia, Curitiba, 10 (4): 641-656.

BATE, C.S. 1888. Report on the Crustacea Macrura collected by H.M.S. Challenger during the years 1873-1876. Zoolo- gy 29. New York, Johnson Reprint, 1965, 942p.

BRUCE, A.J. 1976. Coral reef Caridea and "commensalism". Micronesica, Mangilao, 12 (1): 83-98.

Chace, Jr., F.A. 1972. The shrimps of the Smithsonian-Bredin Caribbean expeditions with a summary of the West Indian shallow water species (Crustacea: Decapoda: Natantia). Smithsonian Contributions to Zoology, Washington, 98: 1-179.

Christoffersen, M.L. 1979. Campagne de la Calypso au large des côtes atlantiques de l'Amérique du Sud (1961-1962). I. 36. Decapod Crustacea: Alpheidae. Resultats Scientifiques des Campagnes de la Calypso, 11: 297-377.

Coelho, P.A.; M.A.C. Santos; M.F.A. Torres; B.R. Monteiro. \& V.A.K. Almeida. 2002. Reino Animália: Filo (ou Subfilo) Crustacea no Estado de Pernambuco, p. 429-482. In: M. TABARELlI \& J.M.C. SiLva (Eds). Diagnóstico da biodiversidade de Pernambuco. Recife, Secretaria de Ciência, Tecnologia e Meio Ambiente, Fundação Joaquim Nabuco, Editora Massangana, 722p.

Collho-Santos, M.A. \& P.A. Coelho. 1994. Porcellanidae (Crustacea, Decapoda, Anomura) do Litoral de Jaboatão dos Guararapes, Pernambuco, Brasil. Trabalhos Oceanográficos da Universidade Federal de Pernambuco, Recife, 23: 177-191.

Duffy, J.E. 1996. Species boundary, specialization, and the radiation of sponge-dwelling alpheid shrimp. Biological Journal of the Linnean Society, Londres, 58: 307-324.

Holthuis, L.B. 1951. A general revision of the Palaemonidae

Revista Brasileira de Zoologia 23 (3): 699-702, setembro, 2006 
(Crustacea, Decapoda, Natantia) of the Americas. I. The subfamilies Euryrhynchinae and Pontoniinae. Allan Hancock Foundation of the University of Southern California Occasional Paper, Los Angeles, 11: 1-331.

Martin, J.W. \& G.E. Davis. 2001. An updated classification of the recent Crustacea. Natural History Museum of Los Angeles County, Science Series, Los Angeles, 39: 1-124.

MeLo, G.A.S. 1996. Manual de identificação dos Brachyura (caranguejos e siris) do litoral brasileiro. São Paulo, Plêiade, FAPESP, 603p.

Ramos-Porto M.R. \& P.A. Coelho. 1998. Malacostraca. Eucarida. Caridea (Alpheoidea excluded), p. 325-350. In: P.S. Young (Ed.). Catalogue of Crustacea of Brazil. Rio de Janeiro, Museu Nacional, XVII+717p.

Rathbun, M.J. 1898. The Brachyura collected by the U.S. Fish Comission Steamer Albatross on the Voyage from Norfolk, Virginia to San Francisco, California, 1887-1888. Proceedings of the United States National Museum, Washington, 21 (1162): 567-616.

Recebido em 13.X.2005; aceito em 17.VIII.2006
Rathbun, M.J. 1900. Results of the Branner-Agassiz Expedition to Brazil. I. The decapod and stomatopod Crustacea. Proceedings of the Washington Academy of Sciences, Washington, 2: 133-156.

Rios, R. \& J.E. Duffy. 1999. Description of Synalpheus williamsi a new species of sponge-dwelling shrimp (Crustacea: Decapoda: Alpheidae), with remarks on its first larval stage. Proceedings of the Biological Society of Washington, Washington, 112: 541-552.

Rocha, L.A.; I.L. Rosa \& B.M. Feitoza. 2000. Sponge-dwelling fishes of Northeastern Brazil. Environmental Biology of Fishes, Dordrecht, 59: 453-458.

Veloso, V.G. 1998. Malacostraca - Eucarida. Porcellanidae, p. 399-405. In: P.S. Young (Ed.). Catalogue of Crustacea of Brazil. Rio de Janeiro, Museu Nacional, XVII+717p.

Veloso, V.G. \& G.A.S. Melo. 1993. Taxonomia e distribuição da família Porcellanidae (Crustacea, Decapoda, Anomura) no litoral brasileiro. Iheringia, Série Zoologia, Porto Alegre, 75: 171-186.

Revista Brasileira de Zoologia 23 (3): 699-702, setembro, 2006 